\title{
Urban Growth Boundaries: An Effective Second-Best Remedy for Unpriced Traffic Congestion?
}

\author{
by \\ Jan K. Brueckner \\ Department of Economics \\ University of California, Irvine \\ 3151 Social Science Plaza \\ Irvine, CA 92697 \\ e-mail: jkbrueck@uci.edu
}

July 2005

\begin{abstract}
This paper evaluates the efficacy of the urban growth boundary (UGB) as a second-best substitute for a first-best toll regime in a congested city. Numerical results show that, while a UGB is welfare improving, validating previous theoretical results, the utility gain it generates is a very small fraction of that achieved under a toll regime. Thus, the UGB is not a useful instrument for attacking the distortions caused by unpriced traffic congestion.
\end{abstract}




\title{
Urban Growth Boundaries: An Effective Second-Best Remedy for Unpriced Traffic Congestion?
}

by

\author{
Jan K. Brueckner*
}

\section{Introduction}

In response to a growing focus on the phenomenon of urban sprawl by the press, policymakers, and the general public, economists have begun to apply the tools of urban economics to analysis of the sprawl issue. The resulting small literature has identified several proximate causes for the rapid spatial expansion of cities, such as investment in an automobile-oriented transportation system (see Glaeser and Kahn (2004) and Nechyba and Walsh (2004)). In addition, the analysis has drawn a distinction between spatial expansion that is warranted on efficiency grounds and expansion that is excessive, being a result of market failures and other distortions that impart an upward bias to urban growth (see Brueckner $(2000,2001)$ ). One such market failure is the failure by developers to account for the potential amenity value of open space around cities, which can lead to excessive development at the urban fringe. Similarly, a failure to account for the externality involved in traffic congestion, which makes the social cost of commuting higher than the private cost, leads to commute trips that are inefficiently long and cities that are excessively spread out.

A favored policy instrument for dealing with urban sprawl is the "urban growth boundary," or UGB, which specifies a city boundary beyond which development may not take place. ${ }^{1}$ As argued by Brueckner (2001), a UGB works perfectly as a restraint on inefficient growth in some circumstances. For example, if the market failure leading to excessive expansion is an overlooked open-space amenity, then the social optimum can be achieved either by a development tax equal to vacant land's amenity value per acre, or by a UGB set at the appropriate distance from the urban center. However, a UGB cannot fully correct some other sprawl-inducing market failures, with the congestion externality being a case in point. To fully correct this distortion, policymakers must instead levy a congestion toll on urban commuters. By raising 
the cost of intracity travel, this toll shrinks the spatial size of the city while greatly increasing central population densities. This densification, which is illustrated in the numerical results of Wheaton (1998), is socially desirable because it limits severe traffic congestion to a relatively small area around the CBD. By contrast, a UGB does not promote central densification to the same extent as a toll regime, limiting its efficacy, although it does address one symptom of market failure by reducing the city's spatial size.

Despite these limitations, a properly chosen UGB is nevertheless welfare-improving in a congested city, as demonstrated in an earlier theoretical literature. This conclusion can be inferred from the results of Kanemoto (1977) and Arnott (1979), who show that the shadow value of land is less than the market value at the city's edge in a laissez-faire equilibrium. The benefit of a UGB as a second-best instrument is more clearly highlighted in the analysis of Pines and Sadka (1985), who extend and synthesize the work of Kanemoto and Arnott.

The purpose of the present paper is to add a quantitative dimension to this earlier secondbest analysis of UGBs. In particular, the paper evaluates the efficacy of the UGB as a policy instrument in a numerical model of a congested city, comparing the welfare gain generated by a UGB to the gain achieved under the first-best toll regime. The results have a number of surprising features, as explained in the ensuing discussion.

The present research was partly inspired by the earlier study of Anas and Rhee (2004), who provide a numerical appraisal of tolls and UGBs in a city that is congested but differs substantially from the standard monocentric model, which was used in the above analyses. Their city has dispersed, instead of centralized, employment, and intracity travel consists of both commuting and shopping trips. In addition, consumer location choices are influenced by random idiosyncratic preferences. The authors' numerical results show that, in such a framework, a congestion-toll regime raises welfare while imposition of a UGB is welfare-reducing. The UGB's harmful impact differs, of course, from the positive impact that arises in the standard model, a clear consequence of the differences in model structures. In carrying out the present research, the goal was to provide a counterpoint to this negative finding by highlighting and quantifying the positive impact of UGBs in the standard model. However, as seen below, the present results convey a message that, in the end, is not too different from that of Anas and 
Rhee.

Section 2 of the paper presents the analytical framework used in the numerical calculations, section 3 presents the results of those calculations, and section 4 offers conclusions.

\section{Analytical Framework}

2a. The setup

The analytical framework relies on the standard model of a congested monocentric city, as developed in many previous papers. It also incorporates several auxiliary assumptions used in the model of Pines and Sadka (1985), as explained below.

Consumers are assumed to have Cobb-Douglas preferences over consumption of housing, denoted $q$ and measured in square feet of floor space, and the nonhousing good $c$, with the utility function given by $v(c, q)=c^{1-\alpha} q^{\alpha}$, where $0<\alpha<1$. Utility is maximized with respect to the budget constraint $c+p q=y-t(x)$, where $p$ is the price per square foot of housing, $y$ is income, and $t(x)$ is commuting cost at distance $x$ from the CBD. Substituting the resulting demand functions back into the utility function, equating the result to a parametric utility level $u$, and solving for $p$ yields $p=\Psi(y-t(x))^{\frac{1}{\alpha}} u^{-\frac{1}{\alpha}}$, where $\Psi$ is a constant. Substituting this housing price function into the demand function for $q$ yields $q=\Gamma(y-t(x))^{\frac{(\alpha-1)}{\alpha}} u^{\frac{1}{\alpha}}$, where $\Gamma$ is a constant.

Housing output, measured in square feet of floor space per unit of land, is given by $\theta S^{\beta}$, where $S$ represents housing capital per unit of land and $0<\beta<1$. Housing developers maximize profit per unit of land, given by $p \theta S^{\beta}-S-r$, where $r$ is rent per unit of land and the price of capital is normalized at unity. Solving the relevant first-order condition for $S$ and substituting $p$ yields $S=\Lambda(y-t(x))^{\kappa} u^{-\kappa}$, where $\kappa=1 / \alpha(1-\beta)$ and $\Lambda$ is a constant. Substituting this solution into the profit function, equating the result to zero, and solving for land rent yields

$$
r=\Omega(y-t(x))^{\kappa} u^{-\kappa} \equiv r(y-t(x), u),
$$

where $\Omega$ is a constant. Finally, noting that population density $D$ equals housing square feet per unit of land divided by square feet per dwelling, it follows that $D=\theta S^{\beta} / q$. Substituting 
the previous solutions,

$$
D=\Phi(y-t(x))^{\kappa-1} u^{-\kappa} \equiv D(y-t(x), u)
$$

where $\Phi$ is a constant.

The city is assumed to be circular, and a fraction $1-\rho$ of the land at each distance is available for housing, with the fraction $\rho$ used for a radial road network. Therefore, the number of residents living beyond a distance $x$ from the CBD is given by

$$
n(x)=\int_{x}^{\bar{x}} 2 \pi s(1-\rho) D(y-t(s), u) d s,
$$

where $\bar{x}$ is the distance to the urban boundary.

With congested travel, the cost per mile of commuting at distance $x$, denoted $T(x)$, depends on the traffic flow across the ring at $x$ (given by $n(x)$ ) relative to the road width at $x$, equal to $2 \pi x \rho$. Adopting the functional form used in much of the prior literature,

$$
T(x)=\eta+\delta\left[\frac{n(x)}{2 \pi x \rho}\right]^{\gamma}
$$

where all parameters are positive.

Differentiating (4) with respect to $n(x)$, the increase in cost per mile at $x$ when another commuter is added to the traffic flow equals $\gamma \delta[n(x) / 2 \pi x \rho]^{\gamma-1}(1 / 2 \pi x \rho)$. Multiplying by $n(x)$, which gives the number of commuters affected, then yields the total damage from the congestion externality at $x$. The congestion toll per mile at $x$, which charges commuters for this damage, is thus given by

$$
\tau(x)=\gamma \delta\left[\frac{n(x)}{2 \pi x \rho}\right]^{\gamma}
$$

Commuting cost from distance $x$, inclusive of the toll, equals the sum of the toll per mile and direct costs per mile across distances inside of $x$. Thus, the function $t(x)$ appearing in the above equations satisfies

$$
t(x)=\int_{1}^{x}[T(s)+\tau(s)] d s .
$$


In a city where no toll is levied, $\tau(x)$ in (5) is set to zero. Note in (6) that the CBD extends out to $x=1$, with commuting cost in its interior equal to zero. ${ }^{2}$

Differentiating (3) and (6) with respect to $x$ yields the following relationships, which play a central role in the numerical exercise:

$$
\begin{aligned}
n^{\prime}(x) & =-2 \pi x(1-\rho) D(y-t(x), u) \\
t^{\prime}(x) & =T(x)+\tau(x) .
\end{aligned}
$$

Eq. (7) indicates that, as $x$ increases, the population outside of $x$ declines at a rate equal to the population residing at $x$. Eq. (8) shows that commuting cost rises with $x$ at a rate equal to the direct cost per mile at $x$ plus the toll at $x$. In addition to (7), the function $n(x)$ satisfies the constraints $n(\bar{x})=0$ and $n(1)=N$, where $N$ is the fixed city population.

In order to conduct a straightforward welfare analysis, the city is assumed to be fully closed, following Pines and Sadka (1985), with an equal share of differential residential land rent accruing to each urban resident as income. ${ }^{3}$ In addition, congestion-toll revenue is redistributed to consumers on an equal per capita basis, possibly reflecting a reduction in other unmodeled taxes. Therefore, letting $r_{a}$ denote agricultural rent, the income parameter $y$ appearing in the equations above must satisfy

$$
\begin{aligned}
y & =y_{\text {exog }}+\frac{1}{N} \int_{1}^{\bar{x}} 2 \pi x(1-\rho)\left[r(y-t(x), u)-r_{a}\right] d x+\frac{1}{N} \int_{1}^{\bar{x}} n(x) \tau(x) d x \\
& \equiv y_{\text {exog }}+y_{\text {rent }}+y_{\text {toll }},
\end{aligned}
$$

where $y_{\text {exog }}, y_{\text {rent }}$ and $y_{\text {toll }}$ are the components of $y$ from exogenous sources, redistributed rents, and redistributed toll revenues, respectively. Note that although $y$ appears explicitly in the first integral in $(9), n(x), T(x)$ and $\tau(x)$ also depend implicitly on this variable. Thus, the level of $y$, set parametrically, affects both $y_{r e n t}$ and $y_{\text {toll }}$, and (9) requires that this level is self-validating in that the components on the RHS add up to $y$ itself. 
An additional condition that is sometimes relevant requires that rent at the edge of the city equals the agricultural rent. This condition is written

$$
r(y-t(\bar{x}), u)=r_{a}
$$

\section{2b. Finding the equilibrium}

In both the laissez-faire and toll-regime cases, the variables $y, u$ and $\bar{x}$ must assume values such that eqs. (1)-(6) and (9)-(10) are satisfied along with the above endpoint constraints on $n(x)$. By contrast, when a UGB is imposed, $\bar{x}$ is set exogenously, and eq. (10) does not apply (the congestion toll $\tau(x)$ is also set at zero).

It is important to note that, in contrast to urban models without congestion, finding the equilibrium is not simply a matter of computing the solution to a set of static simultaneous equations. To see the reason, observe that $T(x)$, commuting cost per mile at $x$, depends from (4) on the distribution of the urban population across all locations in the city, which determines $n(x)$ and thus the traffic flow at $x$. Since $T(x)$ in turn helps determines $t(x)$ and thus population density at $x$ via (2), it follows that density at any one location in the city depends on densities at all other locations. Given this interdependence, the equilibrium must be computed using an iterative procedure that relies on the key equations (7) and (8), which involve the derivatives of the $n$ and $t$ functions. ${ }^{4}$

This procedure works as follows. The city is divided into narrow, discrete rings indexed by $i$, each with a width $\epsilon$, set at a small value. The relationship $x_{i}=1+\epsilon(i-1)$ gives the inner radius of ring $i$, so that ring 1 has inner radius 1 , corresponding to the edge of the CBD. In computing the variables of the model, the distance measure $x$ is replaced by the ring subscript $i$. Thus, $D(y-t(x), u)$ in (2) is replaced by

$$
\left.D_{i}=\Phi\left(y-t_{i}\right)\right)^{\kappa-1} u^{-\kappa}
$$

In addition, (4) is used to write

$$
T_{i}=\eta+\delta\left[n_{i} / 2 \pi x_{i} \rho\right]^{\gamma} .
$$


The variable $n_{i}$ is incremented using recursive relationship

$$
n_{i+1}=n_{i}+\epsilon n^{\prime}\left(x_{i}\right)=n_{i}-\epsilon 2 \pi x_{i}(1-\rho) D_{i}
$$

where the first equality is based on a first-order approximation and the second uses (7) to substitute for $n^{\prime}\left(x_{i}\right)$. Similarly, $t_{i}$ is incremented using

$$
t_{i+1}=t_{i}+\epsilon t^{\prime}\left(x_{i}\right)=t_{i}+\epsilon\left(T_{i}+\tau_{i}\right)
$$

where the second equality uses (8) to substitute for $t^{\prime}\left(x_{i}\right)$. The iterative process starts at $i=1$, with $t_{1}=0$ (indicating no commuting cost from the CBD edge) and $n_{1}=N$. At each iteration, land rent is computed using

$$
r_{i}=\Omega\left(y-t_{i}\right)^{\kappa} u^{-\kappa}
$$

The iterative process is carried out conditional on the values of $y$ and $u$, but these values must be consistent with the achievement of equilibrium, as follows. In the laissez-faire and toll-regime cases, the iterations stop when $i$ reaches a value $i^{*}$ such that $n_{i^{*}} \geq 0$ and $n_{i^{*}+1}<0$, indicating that the population just fits inside an $\bar{x}$ value of $x_{i^{*}}$. Satisfaction of two equilibrium conditions is then checked. First, the value of $r_{i^{*}}$ is compared to $r_{a}$. Second, the value of $y_{\text {exog }}+y_{\text {rent }}+y_{\text {toll }}$ from $(9)$, which has been computed cumulatively in a discrete manner over the sequence of iterations, is compared to assumed value of $y$. If the comparison values diverge by more than the desired degree of accuracy in either case, the values of $y$ and $u$ are adjusted and the iterative process is repeated. ${ }^{5}$

In the UGB case, the iterations stop when $i$ reaches a value $i^{* *}$ such that $x_{i^{* *}}$ equals the specified value of $\bar{x}$. The previous comparison involving $y$ is then carried out, and $n_{i^{* *}}$ is compared to zero. Both $y$ and $u$ are adjusted until the comparison values match with a given degree of accuracy. 


\section{Numerical Results}

This section presents several numerical examples, each of which involves a comparison of three equilibria: the laissez-faire equilibrium, the equilibrium under the toll regime, and the equilibrium with an optimally chosen UGB. The goal is to gauge the efficacy of a UGB by comparing the welfare gain it generates to the gain realized under the first-best toll regime.

The examples rely on a host of assumptions on parameter values, as follows. The housing exponent $\alpha$ in the Cobb-Douglas utility function is set at 0.15 , and the exponent $\beta$ in the housing production function is set at 0.85 (the multiplicative factor $\theta$ is set at 0.0001). ${ }^{6}$ Twenty percent of the land in each ring is devoted to roads, so that $\rho=0.2$. The intercept parameter $\eta$ in the commuting-cost function (4), which is taken to represent the money cost of travel, is set at $\$ 225$, reflecting a $\$ 0.36$ cost per mile (the current Federal allowance), 250 round trips per year, and 1.25 workers per household (as in Bertaud and Brueckner (2005)). The values of the other commuting-cost parameters $(\delta$ and $\gamma)$ differ across the numerical examples, as explained below. Again following Bertaud and Brueckner (2005), agricultural land rent $r_{a}$ is set at $\$ 40,000$ per square mile, reflecting a land value of $\$ 1210$ per acre and a discount rate of 5 percent. The exogenous income value $y_{\text {exog }}$ is set at $\$ 40,000$, a figure approximately equal to US household income, and the city population $N$ is set at 3 million. Finally, the parameter $\epsilon$, which represents ring width, is set at 0.001 miles, a small value intended to achieve a high level of accuracy in identifying the equilibria.

In example 1, the congestion exponent $\gamma$ in (4) is set equal to 1.50, with the multiplicative factor $\delta$ set at $0.000001 .^{7}$ This example is shown in the topmost section of Table 1 , with results for the laissez-faire equilibrium given in the first row. In this equilibruim, the city radius is 22.683 miles, and the utility level is 7771.8997 . The value of $y_{\text {rent }}$ is $\$ 853$, so that total income $y$ equals $\$ 40,835$. Commuting cost at the edge of the city, denoted $\bar{t}$ in the table, is $\$ 7558$, which represents 19 percent of income. Population density $D_{1}$ at the edge of the CBD is 388,861 persons per square mile, and land rent $r_{1}$ at this location is $\$ 357$ million per square mile.

Under the toll regime, the city radius shrinks by about 1.5 miles to 21.158 miles, a decline of about 7 percent. Central density rises by almost a factor of four, to 1,432,612 persons per 
square mile, and central land rent rises by a similar factor. Rental income rises slightly, but income from redistributed tolls equals $\$ 1582$, leading to a notably higher $y$ value of $\$ 42,449$. Commuting cost $\bar{t}$ at the edge of the city, which now includes the toll, rises to $\$ 8112$. In results not shown in the Table, the toll's share in commuting cost per mile (equal to $\tau(x) /[T(x)+\tau(x)]$ ) falls from a high of 59 percent at the CBD's edge to 40 percent at $x=10$ and then to 29 percent at $\bar{x}$, reflecting a decline in congestion moving away from the CBD.

The utility gain under the toll regime is 66.1881 relative to the laissez-faire case. Different approaches can be used to derive the dollar equivalent of this gain, but the following approach seems most natural. In particular, the laissez-faire model is solved holding utility fixed at the first-best level, with $y_{\text {exog }}$ and $y$ adjusted to achieve equilibrium. The resulting increase in yexog tells how much exogenous income would have to rise in the laissez-faire case to generate the utility achieved under the toll regime. For example 1, that income increase is equal to $\$ 335$, or 0.8 percent of $y_{\text {exog }}$.

Before turning to the analysis of the UGB case, consider the laissez-faire and toll-regime equilibria for the remaining examples. Under example 2, shown in the second part of Table 1 , the congestion exponent $\gamma$ is reduced to 1.25 , with $\delta$ raised to 0.00002 . Note that the higher $\delta$ partly offsets the commuting-cost reduction from the decline in $\gamma$. Imposition of the toll regime shrinks the city radius from 23.293 (a larger value than in the first example) to 21.906 miles. Central density and land rent start out lower than before, but their proportional increases under the toll regime are similar to those in the first example. The values of $\bar{t}$ and $y_{\text {toll }}$ are smaller than in example 1, partly reflecting lower tolls. The toll regime raises utility by 31.7010 , a smaller increase than in example 1 . This utility gain is equivalent to a $\$ 158$

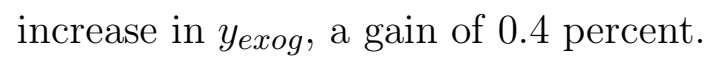

Congestion is reduced further in example 3 , where $\gamma=1.12$ and $\delta=.0001$, and one effect is a larger city radius and a reduction in central density and rent in the laissez-faire case. Imposition of the toll regime raises utility by 21.122, a yet-smaller amount that is equivalent to a $\$ 107$ increase in $y_{\text {exog, }}$, while generating now-familiar changes in the remaining variables. In example 4, the last case considered, $\gamma$ is reduced to 1.00, the value used by Wheaton (1978), while the value of $\delta$ is unchanged. The city's laissez-faire radius rises again, while central 
density and rent are further reduced. The changes under the toll regime again follow previous patterns, but now the utility gain is miniscule, being equal to 1.0372 . This gain is equivalent to an increase in $y_{\text {exog }}$ of only $\$ 5$.

Consider now the UGB cases. For each example, the congestion toll is set at zero, and UGB equilibria are computed for a series of $\bar{x}$ values ranging below the laissez-faire equilibrium value ( $\bar{x}$ is reduced in steps of 0.1 miles). The $\bar{x}$ value in the series associated with the highest utility level is then selected. The typical pattern of utilities resulting from tightening of the UGB is shown in Figure 1, which pertains to example 3.

Turning to the numerical results, the optimal $\bar{x}$ value in example 1 is 18.9 miles, a radius 2.25 miles smaller than under the toll regime. Central density increases only slightly under the optimal UGB, in constrast to the dramatic increase under the toll regime, while $\bar{t}$ falls and $y_{\text {rent }}$ and hence $y$ show slight increases. As noted above, the UGB's failure to foster strong central densification in a congested city limits its efficacy, a conclusion that is dramatically illustrated by the small utility gain under the UGB relative to the laissez-faire case. This increase is only 0.4409, a magnitude that represents only 0.7 percent of the utility gain under the first-best toll regime.

A similar pattern appears in the other examples. In each case, the optimal UGB lies inside the toll-regime's $\bar{x}$, as illustrated in Figure 1 for example 3. In addition, central densification with the UGB is only slight, and the utility increase relative to the laissez-fare case is small. Interestingly, for all the examples, the utility increase ranges between 0.7 and 0.8 percent of the gain under the toll regime. Thus, the results suggest what seems to be a robust conclusion: a UGB is a very ineffective substitute for the first-best toll regime, yielding approximately 1 percent of the utility gain generated by that regime. ${ }^{8}$

Another noteworthy aspect of the results is that the dollar-equivalent welfare gain from the toll regime can be quite small. While the $\$ 335$ gain under example 1 nonnegligible, the $\$ 5$ gain in the less-congested case of example 4 is surprisingly slight, suggesting that the market failure from unpriced traffic congestion in some cases might not be significant enough to warrant our attention. Of course, this conclusion would be overturned if the unitary congestion exponent in example 4 were deemed to be unrealistically low. 
It is worth noting that Wheaton's (1998) calculations yield welfare gains from correcting the congestion externality that are much larger than the present ones. However, several differences between the papers' approaches may make their results noncomparable. First, while Wheaton computes the social optimum for his city, he does not decentralize the optimum via an explicit toll regime. Although redistribution of the toll revenue in the present model should limit any effect from this difference, some effect may remain. Second, land rent in Wheaton's model flows to absentee owners rather than being redistributed to urban residents, and this feature presents an obstacle to straightforward computation of an aggregate welfare gain. Despite these differences in approach, the land-use changes in moving from the laissez-faire equilibrium to the first-best optimal city are similar in both papers.

\section{Conclusion}

This paper has evaluated the efficacy of urban growth boundaries as a second-best remedy for unpriced traffic congestion. The numerical results suggest that a UGB is a very poor substitute for a first-best toll regime, capturing only a tiny fraction of the welfare gain it generates. In this sense, the paper's findings mirror the negative conclusions of Anas and Rhee (1994), which were derived in a nonstandard model. By showing that a UGB reduces welfare, their analysis suggests that policymakers operating in an urban environment like that depicted in their model should shun the UGB as a potential policy instrument. Although a UGB is not harmful in standard monocentric setting, the present results suggest that this instrument is virtually useless in attacking the distortions caused by the congestion externality. Thus, like Anas and Rhee (1994), the implication is that UGBs can be dropped from the list of potential instruments for dealing with unpriced traffic congestion. 
Table 1

\section{Numerical Results}

$\# 1 \quad(\gamma=1.50, \quad \delta=.000001)$

\begin{tabular}{lccccccccc}
\hline \hline & $\bar{x}$ & utility & gain & $y$ & $y_{\text {rent }}$ & $y_{\text {toll }}$ & $\bar{t}$ & $D_{1}$ & $r_{1}$ \\
\hline laissez faire & 22.683 & 7710.8997 & - & $40,835.28$ & 835.28 & 0 & 7558.55 & 388,861 & $3.573 \times 10^{8}$ \\
toll regime & 21.158 & 7777.0878 & 66.1881 & $42,449.13$ & 866.47 & 1582.67 & 8886.81 & $1,432,612$ & $1.368 \times 10^{9}$ \\
best UGB & 18.9 & 7711.3406 & $0.4409(0.7 \%)$ & $40,841.80$ & 841.80 & 0 & 6691.29 & 390,575 & $3.589 \times 10^{8}$ \\
\hline
\end{tabular}

$\# 2 \quad(\gamma=1.25, \quad \delta=.00002)$

\begin{tabular}{lccccccccc}
\hline \hline & $\bar{x}$ & utility & gain & $y$ & $y_{\text {rent }}$ & $y_{\text {toll }}$ & $\bar{t}$ & $D_{1}$ & $r_{1}$ \\
\hline laissez faire & 23.293 & 7840.0825 & - & $40,843.10$ & 843.10 & 0 & 7009.01 & 183,377 & $1.722 \times 10^{8}$ \\
toll regime & 21.906 & 7871.7835 & 31.7010 & $42,085.21$ & 863.83 & 1221.39 & 8112.99 & 575,534 & $5.450 \times 10^{8}$ \\
best UGB & 20.8 & 7840.3000 & $0.2175(0.7 \%)$ & $40,847.51$ & 847.51 & 0 & 6439.94 & 188,026 & $1.728 \times 10^{8}$ \\
\hline
\end{tabular}

$\# 3 \quad(\gamma=1.12, \quad \delta=.0001)$

\begin{tabular}{lccccccccc}
\hline \hline & $\bar{x}$ & utility & gain & $y$ & $y_{\text {rent }}$ & $y_{\text {toll }}$ & $\bar{t}$ & $D_{1}$ & $r_{1}$ \\
\hline laissez faire & 23.426 & 7884.9808 & - & $40,846.56$ & 846.56 & 0 & 6818.73 & 145,911 & $1.341 \times 10^{8}$ \\
toll regime & 22.122 & 7906.6760 & 21.6952 & $42,897.93$ & 863.47 & 1034.45 & 7776.38 & 389,569 & $3.672 \times 10^{8}$ \\
best UGB & 21.4 & 7885.1465 & $0.1657(0.8 \%)$ & $40,850.17$ & 850.17 & 0 & 6356.00 & 146,335 & $1.345 \times 10^{8}$ \\
\hline
\end{tabular}

$$
\# 4 \quad(\gamma=1.00, \quad \delta=.0001)
$$

\begin{tabular}{lccccccccc}
\hline \hline & $\bar{x}$ & utility & gain & $y$ & $y_{\text {rent }}$ & $y_{\text {toll }}$ & $\bar{t}$ & $D_{1}$ & $r_{1}$ \\
\hline laissez faire & 25.200 & 8107.8021 & - & $40,858.34$ & 858.34 & 0 & 5868.49 & 42,820 & $3.936 \times 10^{7}$ \\
toll regime & 24.826 & 8108.8393 & 1.0372 & $41,169.08$ & 861.45 & 307.63 & 6174.65 & 59,173 & $5.481 \times 10^{7}$ \\
best UGB & 24.8 & 8107.8099 & $0.0078(0.8 \%)$ & $40,859.11$ & 859.11 & 0 & 5778.20 & 42,853 & $3.940 \times 10^{7}$ \\
\hline
\end{tabular}


Figure 1: Welfare under a UGB regime (example 3)

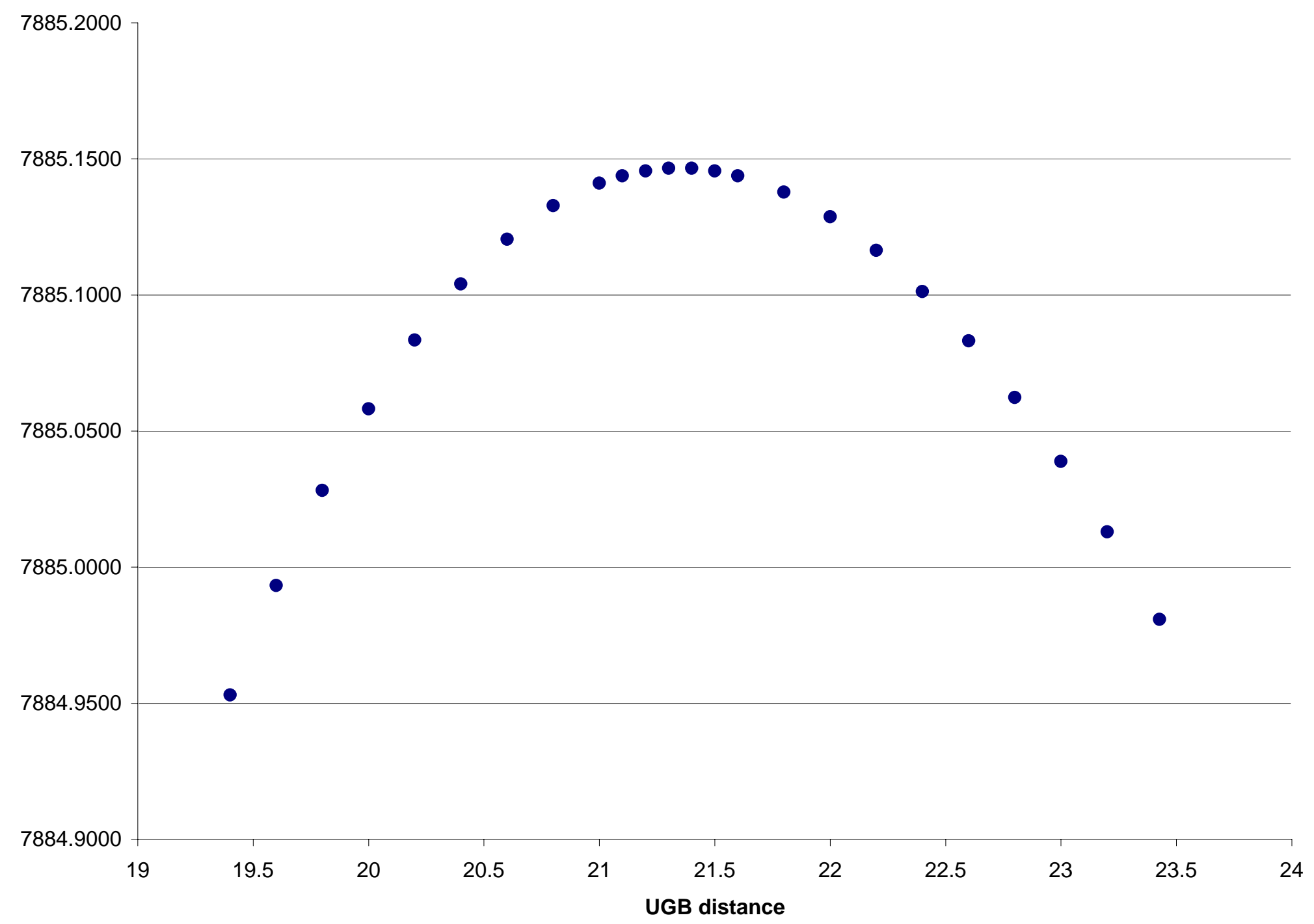




\section{References}

Anas, A., Rhee, H.-J., 2004. Curbing excess sprawl with congestion tolls and urban boundaries. Unpublished paper, State University of New York at Buffalo.

Arnott, R.J., 1979. Unpriced transport congestion. Journal of Economic Theory 21, 294316.

Bertaud, A., Brueckner, J.K., 2005. Analyzing building height restrictions: Predicted impacts and welfare costs, Regional Science and Urban Economics 35, 109-125.

Brueckner, J.K., 2000. Urban sprawl: Diagnosis and remedies. International Regional Science Review 23, 160-171.

Brueckner, J.K., 2001. Urban sprawl: Lessons from urban economics. In: Gale, W.G., Pack, J.R. (Eds.), Brookings-Wharton Papers on Urban Affairs, Brookings Institution, Washington, D.C., pp. 65-89.

Ding, C., KnaAp, G.J., Hopkins, L.D., 1999. Managing urban growth with urban growth boundaries: A theoretical analysis. Journal of Urban Economics 46, 53-68.

Glaeser, E.L., Kahn, M.E., 2004. Sprawl and urban growth. In: Henderson, J.V., Thisse, J.-F. (Eds.), Handbook of Urban Economics, Vol. IV, Elsevier, Amsterdam, forthcoming.

Kanemoto, Y., Cost-benefit analysis and the second land use for transportation. Journal of Urban Economics 4, 483-503.

Nechyba, T.J., Walsh, R., 2004. Urban sprawl. Journal of Economic Perspectives 18(4), $177-200$.

Pines, D., SAdKA, E., 1985. Zoning, first-best, second-best and third-best criteria for allocating land for roads. Journal of Urban Economics 17, 167-183.

Wheaton, W.C., 1998. Land use and density in cities with congestion. Journal of Urban Economics 43, 258-272. 


\section{Footnotes}

*I thank David Pines for helpful discussions in the course of this work and Alex Anas for useful comments on an earlier draft. They are not responsible, however, for any shortcomings in the paper.

${ }^{1}$ See Brueckner $(2000,2001)$ and Ding, Knaap and Hopkins (1999) for institutional discussion regarding the use of UGBs.

${ }^{2}$ This assumption follows Wheaton (1978). The CBD cannot be a point at $x=0$ because the adjacent road width is then zero, implying infinite commuting cost per mile near the CBD under (4).

${ }^{3}$ It is assumed that transport land is acquired by the city at the agricultural rent, so that the differential rent it generates equals zero.

${ }^{4}$ The equilibrium is presumed to be unique, although no proof of this assumption is given. The numerical calculations never suggested the existence of multiple equilibria.

${ }^{5}$ The utility level $u$ is adjusted in increments of .0001 , and at the equilibrium value in the laissez-faire and toll-regime cases, changing $u$ causes $r_{i^{*}}$ to jump from one side of $r_{a}$ to the other. The second requirement is that $y_{\text {exog }}+y_{\text {rent }}+y_{\text {toll }}$ must match $y$ to the second decimal place, a condition that is achieved by appropriate adjustment of $y$ as $u$ is changed. In the UGB equilibrium, changing $u$ causes $n_{i^{* *}}$ to jump from one side of zero to the other, while $y$ is adjusted as above.

${ }^{6}$ The 0.15 value for the parameter $\alpha$, which equals the housing expenditure share, could be viewed as too small. However, this parameter was set to help generate a realistic spatial size for the city. Larger $\alpha$ values yielded an unrealistically large city radius.

${ }^{7}$ An attempt was made to carry out computations for the larger $\gamma$ value of 2.0 , but the adjustment methods for $u$ and $y$ failed in this case (see footnote 5), so that the equilibrium could not be identified.

${ }^{8}$ As discussed by Brueckner (2001), imposition of a UGB when one is not needed reduces welfare. In the present model, if congestion is eliminated by setting $\delta$ equal to zero, and a UGB reduces the city radius by ten percent below its laissez-faire value (as in Table 1), then utility falls by 0.4088 , a small effect. Brueckner (2001) shows that a much larger welfare loss can be generated by a more stringent UGB. 